\title{
Kiel 1969: Ein Erinnerungsort der Geographie
}

\author{
Benedikt Korf $^{1}$ and Ute Wardenga ${ }^{2}$ \\ ${ }^{1}$ Geographisches Institut, Universität Zürich, Winterthurerstraße 190, 8032 Zürich, Schweiz \\ ${ }^{2}$ Leibniz-Institut für Länderkunde, Schongauerstraße 9, 04328 Leipzig, Deutschland
}

Correspondence: Benedikt Korf (benedikt.korf@geo.uzh.ch)

Received: 7 June 2021 - Accepted: 9 August 2021 - Published: 25 August 2021

\begin{abstract}
Kurzfassung. In this editorial, we introduce the special section on the politics of memory of „Kiel 1969“, the famous German geographers' conference, during which, as the myth narrates, a revolution took place within the discipline of German-language geography. By introducing and contextualizing the three individual statements by Julia Verne, Ulf Strohmayer and Peter Weichhart, who all recount their entanglements with the myth of „Kiel 1969“, we invite the reader to reflect upon the dynamics through which „events“ turn into „myths“ that shape individual careers and strategic struggles within the discipline.
\end{abstract}

Kiel 1969. Geographentag. Vorangetrieben durch Fachschaften von Geographischen Instituten der Bundesrepublik Deutschland und unterstützt durch den „GEO Grafiker“ findet eine „Revolution“ in der Geographie statt. So will es eine weitverbreitete Erzählung. Diese Erzählung beschreibt eine Fachgeschichte, in der an einem spezifischen Ort zu einem bestimmten Zeitpunkt (in einem bestimmten Hörsaal) ein revolutionäres Ereignis stattgefunden habe. In Kiel habe die deutschsprachige Geographie gewissermassen einen Paradigmenwandel vollzogen - weg von einer rein beschreibenden Länderkunde hin zu einer wissenschaftlich fundierten Fachwissenschaft. Im Gleichklang damit wurde das, was davor war, als „,reaktionär“, „untheoretisch“, ,essentialistisch“ abgewertet. Für die einen war dies ein längst notwendiger Schritt, für die anderen eine Bedrohung der Einheit des Faches, denn mit der „Verwissenschaftlichung“ wurde auch der Bruch zwischen Physischer Geographie und Humangeographie unausweichlich.

„Kiel 1969“ ist seither zu einem „Erinnerungsort“ der deutschsprachigen Geographie geworden, anhand dessen aktuelle Trends verortet werden. Erinnerungsorte, schreibt der französische Historiker Pierre Nora, sind „Kristallisationspunkte“ eines kollektiven Gedächtnisses (Nora, 1998). Diese Orte, so Nora, haben für eine spezifische Gruppe identitätsstiftende Funktion. Auch Jan Assmann bestätigt diesen Befund, wenn er die „Identitätskonkretheit“ des kulturellen Gedächtnisses hervorhebt: die Überlieferung dieses Wissens sei ,nicht von theoretischer Neugier (Blumenberg) ${ }^{1}$ geleitet" (Assmann, 1988:13), sondern vom Bedürfnis nach sinngeleiteter Identifikation. Zugleich, so Assmann mit Bezug auf Maurice Halbwachs, setze sich die Gegenwart zu ihren Erinnerungsfiguren ,in aneignende, auseinandersetzende, bewahrende und verändernde Beziehung“" (ebd.). Und Aleida Assmann betont, dass sich Bewusstsein generell ,im Zeichen des Abgelaufenen" entwickele und dass sich Erinnerung dann einstelle, ,wenn die Erfahrung, auf die sie sich bezieht, im Rücken liegt“"(Assmann [1999] 2018:11). Erinnerung, könnte man in Anschluss an Hans Blumenberg sagen, ist ,Arbeit am Mythos" (Blumenberg, 1979).

Beim Erinnerungsort „Kiel 1969“ geht es um das kollektive Gedächtnis einer Fachgemeinschaft - der deutschsprachigen Geographie. „Kiel 1969“ ist zum Kristallisationspunkt geworden, anhand dessen immer wieder fachinterne Auseinandersetzungen geführt werden - als notwendiger revolutionärer Schritt gefeiert oder als anhaltende Drohkulisse für den prekär gewordenen Zusammenhalt des Faches beschworen. „Kiel 1969“ als Signum einer revolutionären Wende kann dann auch zu anderen Gelegenheiten ,als Mythos“ aufgerufen werden (vgl. dazu Korf, 2014 und die Beiträge im ersten Teil des Diskussionsforums „Kiel 1969“ in der Geographica Helvetica). Die Aneignung des Mythos erfolgt oft taktisch

\footnotetext{
${ }^{1}$ Assmann bezieht sich hier auf Blumenbergs epochales Werk zur Legitimität der Neuzeit, dessen dritter Teil den Titel trägt: „Der Prozess der theoretischen Neugierte“ (vgl. Blumenberg, 1988:261 ff.).
} 
oder strategisch: ,als Kampfmittel im Zuge der Durchsetzung eigener Ideen“ (Wardenga, 1996:14). Und so haben Erzählungen von und über „Kiel 1969“ oft nur wenig mit dem gemein, was damals ,wirklich geschah“.

So schreiben Steinbrink und Aufenvenne (2017) beispielsweise, man könne bei kulturgeographischen Tagungen im deutschsprachigen Raum heute den Eindruck bekommen, die erste Tagung zur „Neuen Kulturgeographie“, 2004 in Leipzig abgehalten, ,,sei das neue Kiel!“ (Steinbrink und Aufenvenne, 2017:84). „Leipzig“ werde als paradigmatischer Wendepunkt in der Humangeographie stilisiert - als ,Leipziger Wende?“(ebd.). Auch in Leipzig, wie in Kiel 1969 sozusagen, hätten sich ,junge Wilde“ daran gemacht, die „Humangeographie zu revolutionieren“ (Berndt und Pütz, 2007:7). Die Arbeit am „Mythos Kiel“ findet also im alltäglichen „Geographiemachen“ der Disziplin immer wieder statt - und sie dient der internen Positionierung und Absicherung.

2019 fand der Deutsche Kongress für Geographie (DKG) wieder in Kiel statt - „50 Jahre danach“. Für uns diente dies als Anlass, auf das Ereignis „Kiel 1969“ zurückzuschauen und seine Funktion als Erinnerungsort ins Gedächtnis zu rufen. Dabei ist, wie Jan Assmann festhält, zwischen individuellem Gedächtnis und „kommunikativem“ Gedächtnis als geteilte kulturelle Praxis zu unterscheiden. Für eine Podiumsdiskussion auf dem DKG in Kiel 2019 fragten wir drei Geographinnen und Geographen aus unterschiedlichen Generationen an, ihr individuelles Gedächtnis in Erinnerung zu rufen, um über den Erinnerungsort „Kiel 1969“ nachzudenken.

Wir fragten sie: Welche Erzählung von Kiel war für sie wichtig? Welche Rolle spielt diese Erzählung in ihrer eigenen Biographie? Und welchen Einfluss hat der Erinnerungsort „Kiel 1969“ in disziplinären Auseinandersetzungen? Indem sie ihre individuellen Erinnerungen auf dem Podium darlegten, trugen sie zugleich dazu bei, den Erinnerungsort „Kiel 1969“ als Teil des kulturellen Gedächtnisses des Faches Geographie zu kartieren und zur kritischen Diskussion zu stellen.

Ute Wardenga leitete das Podiumsgespräch aus disziplinhistorischer Sicht ein, indem sie die Kieler Geschehnisse nochmals Revue passieren ließ und ein neues Geschichtsbild entwarf (vgl. ausführlich Wardenga, 2020). Danach handelte es sich bei „Kiel 1969“ um eine „Revolution mit Ansage“ (Wardenga, 2021). Denn in die Vorbereitung der berühmtberüchtigten Diskussionsveranstaltung (vgl. Meckelein und Borcherdt, 1970:191-207 und 208-232) waren alle wichtigen Stakeholder der Geographie einbezogen. Allerdings war die Mehrheit der Professorenschaft außerstande, die sich bereits weit vor „Kiel“" abzeichnenden Krisenerscheinungen angemessen zu interpretieren. Ein wesentlicher Grund für dieses Unvermögen lag nach Wardenga darin, dass aus den Systemkonkurrenzen des Kalten Krieges enorme Modernisierungsanforderungen erwuchsen.

Diese Modernisierungsanforderungen erzwangen eine grundsätzliche Umstellung von Temporalitätsregimen, mit denen das Fach arbeitete. Aus einem bis dato historisch mit der Leitunterscheidung Vergangenheit/Gegenwart operierenden Fach wurde eine stärker fortschrittsorientierte Disziplin, die mit der Leitunterscheidung Gegenwart/Zukunft zu forschen begann (vgl. dazu Wardenga, 2020:2 ff.). Der Kieler Geographentag war deshalb ein Ort hoher wissenschaftssozialer Verdichtung, weil er für eine breite Fachöffentlichkeit erstmals die Notwendigkeit dringend anstehender Reformen erlebbar machte und zugleich in erschütternder Weise zeigte, dass die vorwiegend mit dem Paradigma der Länderund Landschaftskunde arbeitende Professorenschaft hierzu schwerlich in der Lage sein würde.

Auf Wardenga folgten Beiträge von Julia Verne, einer Repräsentantin der jüngeren Generation, die „Kiel 1969“ nur noch vom Hörensagen kennt, und zugleich dafür plädierte, gegenwärtige Theoriedebatten mit einer stärkeren historischen Tiefenschärfe zu führen; Ulf Strohmayer, der aus seinen eigenen Studienerfahrungen an der TU München zu Beginn der 1980er Jahre erzählte und die dort herrschende Resistenz gegenüber den Impulsen aus „Kiel 1969“ beklagte, die ihn schließlich dazu motivierten, seine akademische Karriere außerhalb Deutschlands weiterzuverfolgen; und Peter Weichhart, der damals schon Student war und von einem seiner akademischen Lehrer den erschütterten Bericht von einem angeblichen studentischen „Putschversuch“ erhielt.

Verne betonte, dass sie in die deutschsprachige Geographie über die „Neue Kulturgeographie“ sozialisiert wurde, an der sie sich zugleich ,immer wieder gerieben“ habe. Doch geschah dies erst im Promotionsstudium und nach einem Ausflug in die anglophone New Cultural Geography. Zum Zeitpunkt ihres Diplomstudiums hätten noch Handlungstheorie und Münchner Schule die zentrale Rolle gespielt. Dort sei „Kiel 1969“ als „unglaublich wichtiger Moment“ angeklungen. In ihrer Generation werde „Kiel 1969“ jedoch nur noch als einer unter einer Reihe von Momenten der Veränderung im Fach wahrgenommen (Verne, 2021:160).

Mit der Inflation der Rezeption neuer Theorien in den letzten zwei Jahrzehnten sei „Veränderung“ zur eigentlichen Konstante in der deutschsprachigen Humangeographie geworden: von Handlungstheorie zur Diskurstheorie, zur Affekttheorie und zum New Materialism und so weiter. Für Verne bieten sich in dieser neuen Vielfalt interessante Anschlussmöglichkeiten, nicht nur mit Nachbardisziplinen, sondern auch mit der Historiographie der Geographie. Zugleich könnte man ergänzen, dass sich auch eine neue Dynamik im Fach ausgebildet hat: von einer „Tendenz zur Schulbildung“, die Strohmayer noch 1998 beobachtet hat (Strohmayer, 1998:103), zu einer „Anfälligkeit für Modeerscheinungen“, die vor allem über die anglophone Geographie in die deutschsprachige Geographie diffundiert.

Strohmayers Studienzeit lag zeitlich noch sehr viel näher an „Kiel“. Aber bereits in seiner Studienzeit Ende der 1970er und Anfang der 1980er Jahre ist Strohmayer „Kiel 1969“ nur als ,indirekter, vermittelter und praktizierter Betrachtungspunkt" (Strohmayer, 2020:216) begegnet: insbesondere in 
der funktionalistischen Ausrichtung auf praktische Relevanz. Unter den herrschenden „Leitwölfen“" war das Institut an der TU München ,weitgehend eine theorieferne Zone“ (Strohmayer, 2020:217), doch gab es auf ,weniger sichtbaren Posten“ begrenzte Freiräume für neue Ansätze und Theorien, auch gesellschaftskritischer Art. Strohmayer ruft hier Heiner Dürr in Erinnerung, der ein offenes Ohr gehabt habe.

Doch stellt sich aus dieser Hierarchisierung - theorieferne Leitwölfe gegen theorieaffine Randfiguren - für Strohmayer die „Organisationsfrage“, wie man im Geist von „Mai 1968“ formuliert habe: Wie waren (und sind!) die Strukturen an deutschen Hochschulen beschaffen, dass sie einem offenen Dialog ,diametral“ entgegenstehen - durch die Prekarisierung des wissenschaftlichen Nachwuchses. Strohmayer, der seit Jahrzehnten im anglophonen Hochschulsystem tätig ist, muss es wissen: implizit führt er die stärkere Radikalität der Neuerungen der anglophonen Geographie auf die Strukturen der dortigen Hochschulen zurück, wo tenure schon kurz nach der Promotion erreicht werden kann, wodurch intellektuelle Unabhängigkeit schon früh auch institutionell abgebildet wird.

Weichhart studierte zum Zeitpunkt von „Kiel 1969“ bereits im fünften Semester, nahm aber nicht am Kieler Geographentag teil. Aber einer seiner Professoren „berichtete“ eher erschüttert von dortigen revolutionären Umtrieben, die zum Glück von den etablierten Fachvertretern als Unfug entlarvt worden seien. Gerade dies machte Weichhart neugierig und so wurde „Kiel 1969“, insbesondere die von Bartels und Hard eingeforderte wissenschaftstheoretische Grundlegung, zu einer wichtigen Konstante seiner akademischen Sozialisation.

Doch fand er für dieses Interesse an (Wissenschafts-) Theorie über lange Zeit wenig Resonanz an seinen Wirkungsorten: weder an der LMU, noch in Salzburg: „Kiel ist an der damaligen Salzburger Geographie eigentlich spurlos vorübergegangen“, hält Weichhart fest und ergänzt, für seine Kolleginnen und Kollegen in Salzburg habe ,theoretisch“ einfach nur „unwirklich“ bedeutet (Weichhart, 2021:26). Weichhart, der die Länderkunde eher ,öde“ fand, bezog seine theoretischen Inspirationen daher aus seinen Nebenfächern Germanistik und Philosophie.

Für Weichhart ist „Kiel 1969“ aber auch in der jüngeren Generation eine ,weitgehend vergessene“ Geschichte. Zugleich warnt er davor, die Fundamentalkritik aus „Kiel 1969“ an der klassischen Geographie einfach ungeprüft zu übernehmen: die klassische Geographie sei ,,keineswegs ,theorielos“", sondern man finde dort durchaus ,ernsthafte und komplexe Ansätze einer theoretischen Reflexion“ (Weichhart, 2021:25). Das Problem sei, dass heute über die klassische Geographie nur noch vom Hörensagen berichtet werde: Viele hätten anscheinend die Klassiker ,nicht allzu häufig im Original gelesen“( (ebd.) ${ }^{2}$.

\footnotetext{
${ }^{2}$ Weichharts Aussage sei hier, auf Anregung eine*r Gutachter*in, dahingehend ergänzt (oder relativiert), dass auch zeitgenös-
}

In der sich daran anschließenden Diskussion meldeten sich viele Hochschulgeographen (fast ausnahmslos Männer) zu Wort, die nach „Kiel 1969“ an deutschen Hochschulen gelehrt haben. Einigen ging die kritische Aufarbeitung nicht weit genug: Die Nazivergangenheit vieler Professoren sei unterbelichtet geblieben (vgl. dazu z. B.: Fahlbusch et al., 1989; Sandner, 1983). Andere sahen durchaus Orte, an denen die Aufbruchsstimmung von Kiel weitergetragen worden sei meist jedoch am Rande des Mainstreams. So habe es zum Beispiel in der Geographischen Entwicklungsforschung mit der Rezeption der Dependenztheorie Räume für kritische Gesellschaftstheorie gegeben, doch seien solche Initiativen eben am Rande der Maschinerie der Disziplin verblieben. Erst mit der „Neuen Kulturgeographie“ sei Theoriearbeit satisfaktionsfähig geworden.

Andere Diskussionsbeiträge betonten hingegen stärker den pragmatischen Erfolg der Geographie als Berufsmaschinerie im Planungsbereich, die dem Fach über die akademische Welt hinaus Einfluss gebracht habe und für deren Erfolg „Theorie“ und „Kritik“ keine hilfreichen Kategorien seien (vgl. zur damaligen Diskussion: Wardenga et al., 2011). Sie bestätigen damit indirekt das Bild der Hochschulgeographie, das Strohmayer auf dem Podium für seine Studienzeit an der TUM gezeichnet hatte. Auch später noch, in Vernes Studium, wurde „Kiel 1969“ mit der Forderung nach Praxisbezug in Verbindung gebracht (etwas, von dem Verne selbst sich eher ,befreien“ wollte, vgl. Verne, 2021:159).

Das Podiumsgespräch verhandelte damit nicht nur die Erinnerungspolitik und Gedächtniskommunikation zu „Kiel 1969“ in der deutschsprachigen Geographie, sondern warf zugleich grundsätzlichere epistemologische Fragen zur geographischen Theoriegenese auf. Denn es ist auffallend, dass sich auf ,die Alten“ immer in pejorativer Absicht bezogen wird - ein Punkt, den auch Verne und Weichhart in ihren Beiträgen ansprechen. Die Geschichte der Disziplin scheint nicht etwas zu sein, zu dem man sich positiv positioniert und deren Texte man mit Gewinn liest.

Mit anderen Worten: die Geographie hat keinen Kanon anerkannter „Heldenfiguren“ - die Helden, das sind immer die heutigen $^{3}$. Ein Alfred Hettner ist kein Max Weber. Die Geographie hat keine Gründerfiguren, wie sie die Soziologie in ihren „Klassikern“ hat, deren Gedanken sie sich immer wieder in kritisch-apologetischer Theoriearbeit aneignet. Aneignung des Vergangenen findet in der Geographie vorwiegend durch ein Othering statt. Klassiker lesen scheint dabei keine Tugend zu sein, denn für eine strategic canonization (Barnes, 2015) scheint das Hörensagen zu reichen.

Oralität befördert Mythenbildung - wie Wardenga (2021) in diesem Themenheft am Beispiel des Kieler Geographen-

sische Theoretiker*innen - man denke an Foucault oder Latour nur selten ausführlich „,im Original“ gelesen werden . .

${ }^{3}$ Vgl. dazu die Diskussion in der anglophonen Geographie zur Frage, warum es im Fach keinen Kanon gibt und ob das ein Problem ist, insbesondere Barnes (2015), Barry (2015), and Powell (2015). 
tags von 1969 zeigt. Dabei erfüllen Mythen eine wichtige wissenschaftssoziale Funktion. Denn solche Geschichten können „im Medium von Erzählungen [...] die soziale Welt [... $]$ strukturieren“. Dadurch helfen sie, „Sinnordnungen zu schaffen, Identitäten zu formen, Legitimationsansprüche durchzusetzen und orientieren so das Handeln in einer jeweiligen Gegenwart" (Wardenga, 2011:7). Der Vorteil mythologisch geformter Erzählungen besteht dabei darin, dass sie ,die klaffende Lücke von Schein und Sein durch symbolische Ersatzleistung“" verdecken, weil Mythen im milderen „als ob der Vorgeblichkeit“ (Wardenga, 2011:11) operieren und unter deutlicher Parteinahme gegen das Herkommen und für eine jeweilige Gegenwart sprechen.

Der daraus resultierende starke Bezug zur Gegenwart bringt aber auch Nachteile mit sich. Denn die mythologische Narrationsstruktur verführt notwendigerweise dazu, die je eigene Gegenwart mit ihren spezifischen Werten und Vorstellungen unreflektiert absolut zu stellen. Das kann in der Praxis des Geschichten Erzählens über Geographie und Geograph*innen dann dazu verleiten, dass die Vergangenheit zugunsten gegenwärtiger Legitimationsbedürfnisse (unbewusst) koloni(ali)siert wird.

„Arbeit am Mythos“ heißt deshalb nicht zuletzt: Lernen, mit unbequemen, ja manchmal sogar erschreckenden Differenzen und Weltvorstellungen vergangener Gegenwarten umzugehen, z. B. auch in Gestalt anderer Formen des doing geographies. Vielleicht würde als Remedium gegen allfällige pejorative Bezugnahmen auf die Altvorderen die paradoxe Einsicht helfen, dass sich Wissenschaftsdisziplinen vorwiegend durch Wandel auf Konstanz stellen, auch und gerade dann, wenn - wie in Kiel 2019 - außergewöhnliche Ereignisse als Orte kollektiver Erinnerungen rekonfiguriert werden.

Haftungsausschluss. Publisher's note: Copernicus Publications remains neutral with regard to jurisdictional claims in published maps and institutional affiliations.

Begutachtung. This paper was edited by Nadine Marquardt and reviewed by one anonymous referee.

\section{Literatur}

Assmann, A.: Erinnerungsräume, Formen und Wandlungen des kulturellen Gedächtnisses, C. H. Beck, München, [1999] 2018.

Assmann, J.: Kollektives Gedächtnis und kulturelle Identität, in: Kultur und Gedächtnis, Herausgeber: Assmann, J. und Hölscher, T., Suhrkamp, Frankfurt am Main, 9-19, 1988.

Barnes, T.: Afterword: strategic canonization?, J. Hist. Geogr., 49, 94-95, 2015.

Barry, A.: Geography and other disciplines: genealogy, anamnesis and the canon, J. Hist. Geogr., 49, 85-93, 2015.

Berndt, C. und Pütz, R.: Kulturelle Geographien nach dem Cultural Turn, in: Kulturelle Geographien: Zur Beschäftigung mit Raum und Ort nach dem Cultural Turn, Herausgeber: Berndt, C. und Pütz, R., Transcript, Bielefeld, 7-25, 2007.

Blumenberg, B.: Arbeit am Mythos, Suhrkamp, Frankfurt am Main, 1979.

Blumenberg, B.: Die Legitimität der Neuzeit, erneuerte Auflage, Suhrkamp, Frankfurt am Main, 1988.

Fahlbusch, M., Rössler, M., und Siegrist, D.: Geographie und Nationalsozialismus - 3 Fallstudien zur Institution Geographie im Deutschen Reich und in der Schweiz, Urbs et Regio 51, Kassel, 1989.

Korf, B.: Kiel 1969 - ein Mythos?, Geogr. Helv., 69, 291-292, https://doi.org/10.5194/gh-69-291-2014, 2014.

Meckelein, W. und Borcherdt, C.: Deutscher Geographentag Kiel, 21. bis 26. Juli 1969, Tagungsbericht und wissenschaftliche Abhandlungen, Steiner, Wiesbaden, 1970.

Nora, P.: Zwischen Geschichte und Gedächtnis, Fischer Taschenbuch, Frankfurt am Main, 1998.

Powell, R.: Notes on a geographical canon? Measures, models and scholarly enterprise, J. Hist. Geogr., 49, 2-8, 2015.

Sandner, G.: Die „Geographische Zeitschrift“ 1933-1944. Eine Dokumentation über Zensur, Selbstzensur und Anpassungsdruck bei wissenschaftlichen Zeitschriften, Geogr. Z., 71, 65-87, 127149, 1983.

Steinbrink, M. und Aufenvenne, P.: Über Othering, Entgrenzung und Mainstreamisierung der Neuen Kulturgeographie. Eine kleine Disziplinbeobachtung, Mitt. Österreich. Geogr. Gesell., 159, 83-104, https://doi.org/10.23781/moegg159-083, 2017.

Strohmayer, U.: Methodisches Denken im Poststrukturalismus, Geogr. Helv., 53, 103-106, https://doi.org/10.5194/gh-53-1031998, 1998.

Strohmayer, U.: An was genau erinnert „Kiel 1969“? , Geogr. Helv., 75, 215-219, https://doi.org/10.5194/gh-75-215-2020, 2020.

Verne, J.: „Kiel 1969“ - ein Erinnerungsort, Geogr. Helv., 76, 159161, https://doi.org/10.5194/gh-76-159-2021, 2021.

Wardenga, U.: Geographie als Chorologie - Alfred Hettners Versuch einer Standortbestimmung, in: 100 Jahre Geographie an der Ruprecht-Karls-Universität Heidelberg (1895-1995), Herausgeber: Barsch, D., Fricke, W., und Meusburger, P., Geographisches Institut der Universität Heidelberg, Heidelberg, 1-17, 1996.

Wardenga, U.: Geographie als Brückenfach - oder Arbeit am Mythos, entgrenzt, studentische Zeitschrift für Geographisches, Ausgabe 1, SoSe 2011, 5-16, online aufrufbar: http: //entgrenzt.de/ausgaben/entgrenzt-ausgabe-1/ (letzter Zugriff: 24. August 2021), 2011.

Wardenga, U.: Vergangene Zukünfte - oder: Die Verhandlung neuer Möglichkeitsräume in der Geographie, Geogr. Z., 108, 4-22, https://doi.org/10.25162/gz-2019-0009, 2020.

Wardenga, U.: Kiel 1969: Ein quellenkritischer Blick auf Tradierungsprozesse als „Arbeit am Mythos“, Geogr. Helv., 76, 299303, https://doi.org/10.5194/gh-76-299-2021, 2021.

Wardenga, U., Henniges, N., Brogiato, H. P., und Schelhaas, B.: Der Verband Deutscher Berufsgeographen 1950-1979: Eine sozialgeschichtliche Studie zur Frühphase des DVAG, Forum IfL 16, Leibniz-Institut für Länderkunde, Leipzig, S. 136, 2011.

Weichhart, P.: Kiel 1969 als Erinnerungsort der deutschsprachigen Geographie - einige autoethnographische Anmerkungen, Geogr Helv., 76, 23-28, https://doi.org/10.5194/gh-76-23-2021, 2021. 